\title{
Properties of beta oscillations in Dup15q syndrome
}

Vidya Saravanapandian ${ }^{1 *}$ (D, Joel Frohlich ${ }^{1,2,3}$, Joerg F. Hipp ${ }^{2}$, Carly Hyde ${ }^{1}$, Aaron W. Scheffler ${ }^{4}$, Peyman Golshani ${ }^{5,6}$, Edwin H. Cook ${ }^{7}$, Lawrence T. Reiter ${ }^{8}$, Damla Senturk ${ }^{4}$ and Shafali S. Jeste ${ }^{1}$

\begin{abstract}
Background: Duplications of 15q11.2-q13.1 (Dup15q syndrome) are highly penetrant for autism, intellectual disability, hypotonia, and epilepsy. The 15q region harbors genes critical for brain development, particularly UBE3A and a cluster of gamma-aminobutyric acid type $A$ receptor $\left(G A B A_{A} R\right)$ genes. We recently described an electrophysiological biomarker of the syndrome, characterized by excessive beta oscillations $(12-30 \mathrm{~Hz})$, resembling electroencephalogram (EEG) changes induced by allosteric modulation of $G_{A B A}{ }_{A} R s$. In this follow-up study, we tested a larger cohort of children with Dup $15 q$ syndrome to comprehensively examine properties of this EEG biomarker that would inform its use in future clinical trials, specifically, its (1) relation to basic clinical features, such as age, duplication type, and epilepsy; (2) relation to behavioral characteristics, such as cognition and adaptive function; (3) stability over time; and (4) reproducibility of the signal in clinical EEG recordings.

Methods: We computed EEG power and beta peak frequency (BPF) in a cohort of children with Dup15q syndrome ( $N=41$, age range 9-189 months). To relate EEG parameters to clinical (study 1) and behavioral features (study 2), we examined age, duplication type, epilepsy, cognition, and daily living skills (DLS) as predictors of beta power and BPF. To evaluate stability over time (study 3), we derived the intraclass correlation coefficients (ICC) from beta power and BPF computed from children with multiple EEG recordings ( $N=10$, age range 18-161 months). To evaluate reproducibility in a clinical setting (study 4), we derived ICCS from beta power computed from children ( $N$ = 8, age range 19-96 months), who had undergone both research EEG and clinical EEG.

Results: The most promising relationships between EEG and clinical traits were found using BPF. BPF was predicted both by epilepsy status $\left(R^{2}=0.11, p=0.038\right)$ and the DLS component of the Vineland Adaptive Behavior Scale $\left(R^{2}\right.$ $=0.17, p=0.01$ ). Beta power and peak frequency showed high stability across repeated visits (beta power ICC $=$ 0.93 , BPF ICC $=0.92$ ). A reproducibility analysis revealed that beta power estimates are comparable between research and clinical EEG $(I C C=0.94)$.

(Continued on next page)
\end{abstract}

\footnotetext{
* Correspondence: vidyasaravanapandian@gmail.com

${ }^{1}$ Center for Autism Research and Treatment, Semel Institute for

Neuroscience, University of California Los Angeles, Los Angeles, CA 90024,

USA

Full list of author information is available at the end of the article
}

(C) The Author(s). 2020 Open Access This article is licensed under a Creative Commons Attribution 4.0 International License, which permits use, sharing, adaptation, distribution and reproduction in any medium or format, as long as you give appropriate credit to the original author(s) and the source, provide a link to the Creative Commons licence, and indicate if changes were made. The images or other third party material in this article are included in the article's Creative Commons licence, unless indicated otherwise in a credit line to the material. If material is not included in the article's Creative Commons licence and your intended use is not permitted by statutory regulation or exceeds the permitted use, you will need to obtain permission directly from the copyright holder. To view a copy of this licence, visit http://creativecommons.org/licenses/by/4.0/ The Creative Commons Public Domain Dedication waiver (http://creativecommons.org/publicdomain/zero/1.0/) applies to the data made available in this article, unless otherwise stated in a credit line to the data. 


\begin{abstract}
(Continued from previous page)
Conclusions: In this era of precision health, with pharmacological and neuromodulatory therapies being developed and tested for specific genetic etiologies of neurodevelopmental disorders, quantification and examination of mechanistic biomarkers can greatly improve clinical trials. To this end, the robust beta oscillations evident in Dup15q syndrome are clinically reproducible and stable over time. With future preclinical and computational studies that will help disentangle the underlying mechanism, it is possible that this biomarker could serve as a robust measure of drug target engagement or a proximal outcome measure in future disease modifying intervention trials.
\end{abstract}

Keywords: Dup15q syndrome, Autism, Biomarkers, EEG, GABA, UBE3A, Neurodevelopmental disorders

\section{Background}

Genetic testing for neurodevelopmental disorders (NDDs) has become increasingly precise and clinically available. As a result, hundreds of causative genetic etiologies for NDDs have now been identified, from single gene mutations to copy number variants [1]. Under a conceptual framework of precision health for NDDs, identification of mechanistic biomarkers that reflect specific genetic disruptions can greatly improve clinical trials for these genetic syndromes by serving as measures of drug target engagement, stratification, or as outcome measures that precede more subtle, yet meaningful, behavioral responses to treatment $[2,3]$.

Recently, we quantified a robust electroencephalography (EEG) biomarker of the copy number variant syndrome caused by duplications of chromosome 15q11.2-q13.1 (Dup15q syndrome) [4-6]. Dup15q syndrome is highly penetrant for autism spectrum disorder (ASD), accounting for $1-3 \%$ of cases [7, 8]. Individuals with this syndrome also have comorbid global developmental delay, intellectual disability (ID), hypotonia, and a high rate of epilepsy [6, 9-11]. Based on allelic inheritance, the $15 \mathrm{q}$ region harbors several genes critical for brain development and synaptic function, particularly $U B E 3 A$, and a cluster of bi-allelically expressed gammaaminobutyric acid type A receptor $\left(\mathrm{GABA}_{\mathrm{A}} \mathrm{R}\right)$ genes, GABRB3, GABRA5, and GABRG3, which encode $\beta 3$, $\alpha 5$, and $\gamma 3$ subunits, respectively [10]. UBE3A encodes a ubiquitin protein ligase and is maternally expressed (i.e., paternally imprinted) in most neurons [12, 13] while playing an important role in regulating synaptic development and function [12, 14]. Functional loss of the $U B E 3 A$ protein causes Angelman syndrome, another rare genetic NDD whose clinical features (e.g., ID and epilepsy) and etiology (e.g., UBE3A dysfunction) [15] partially overlap with Dup15q syndrome. However, the beta EEG phenotype found in cases of maternal Dup15q syndrome is also seen in paternal duplications, in which $U B E 3 A$ is minimally impacted [5]. This suggests a crucial role for nonimprinted $15 \mathrm{q}$ genes, rather than $U B E 3 A$, in generating the EEG phenotype.
Different types of duplications result in Dup15q syndrome. Interstitial duplications generally result in one extra copy (i.e., partial trisomy) of the $15 \mathrm{q}$ region that remains on the same chromosome arm as the original copy. In some cases, interstitial triplications occur as two extra copies (i.e., partial tetrasomy) of the $15 \mathrm{q}$ region. Isodicentric duplications result in two extra maternal copies of the $15 \mathrm{q}$ region manifesting as a supernumerary chromosome [10]. Individuals with interstitial duplications tend to have a milder clinical phenotype and lower incidence of epilepsy compared to those with isodicentric duplications, implying a gene dosage effect $[6,7,16]$ on clinical outcomes.

Spontaneous, high amplitude beta $(12-30 \mathrm{~Hz})$ oscillations represent an EEG biomarker of Dup15q syndrome [4-6]. This beta EEG phenotype was first noted in a comprehensive case series of children with interstitial duplications, based on clinical EEGs obtained for epilepsy monitoring $[6,17]$. Our group then quantified this EEG phenotype in high-density research EEG recordings and found that beta oscillations significantly distinguish children with Dup15q syndrome from age-matched typically developing children and age- and cognitively matched children with nonsyndromic ASD and ID [4]. Evidence from clinical and preclinical studies demonstrates a crucial role for GABAergic neurotransmission in the generation of beta oscillations, thus implicating the GABAergic system in the Dup15q syndrome beta EEG phenotype. Positive allosteric modulators of $\mathrm{GABA}_{\mathrm{A}} \mathrm{Rs}$ (e.g., benzodiazepines) induce beta oscillations in human scalp recordings and intracranial recordings from rodents [18-21]. These pharmacological agents enhance the inhibitory chloride current through the $\mathrm{GABA}_{\mathrm{A}} \mathrm{R}$ when bound in the presence of GABA [22]. Conversely, blockade of $\mathrm{GABA}_{\mathrm{A}} \mathrm{Rs}$ results in desynchronization and diminished oscillatory power at high frequencies in the beta/gamma $(12-80 \mathrm{~Hz})$ range [23]. Furthermore, cases of Angelman syndrome caused by deletions of 15q11-q13 (the genetic converse of Dup15q syndrome) demonstrate lower EEG beta power as compared with etiologies not involving the 
$\mathrm{GABA}_{\mathrm{A}} \beta 3 / \alpha 5 / \gamma 3$ gene cluster [24], also suggesting that beta power may serve as a biomarker of altered GABA neurotransmission.

Although an exact mechanism of beta oscillations has not been elucidated in Dup15q syndrome, increased gene dosage of GABRB3, GABRA5, and GABRG3 [16, 25-27] suggests dysfunctional GABAergic neurotransmission. While the foregoing genes are likely crucial to the presence of the beta EEG phenotype [5], UBE3A plays a critical role in the development and function of GABAergic circuits, including neurons that co-release GABA in rodents $[28,29]$, suggesting that it also affects beta oscillations. As further evidence of the intimate connection between $U B E 3 A$ and GABA, the $\mathrm{GABA}_{\mathrm{A}}$ enhancers gaboxadol and ganaxolone have been shown to restore behavioral phenotypes in Ube3a knockout mice $[30,31]$. Thus, UBE3A overexpression likely affects GABAergic transmission in this syndrome. Future studies focusing on quantifying beta oscillations in $U B E 3 A$ overexpressed mouse models will help elucidate the exact relationship between $U B E 3 A$ and beta oscillations in Dup15q syndrome.

As disease modifying therapies, particularly those that modulate altered GABA signaling, are developed and tested in Dup15q syndrome, the EEG biomarker in this syndrome has the potential to serve as a measure of drug target engagement, stratification, or as a proximal outcome measure. In order to facilitate and inform the use of this biomarker in clinical trials, we examined the following properties in a large cohort of children with this syndrome: (1) relation to clinical features, including age, duplication type, and epilepsy; (2) relation to behavior, namely those features that contribute most to the clinical heterogeneity of the syndrome (cognition and adaptive skills); (3) stability over time; and (4) reproducibility of the signal in clinical EEG. Given that this work spanned several years and projects, we also had the opportunity to compare results generated from different EEG data preprocessing pipelines, thus indirectly testing the reproducibility of the biomarker across analytic pipelines. To accomplish these goals and to adequately enhance our clinically representative sample size in this rare disorder, we partnered with a patient advocacy group, the Dup15q Alliance, and we collected EEG data at two consecutive national family meetings, as well as at our own institution. This study reflects an effort to improve clinical trial readiness in this genetic syndrome by comprehensively characterizing the aspects of this biomarker that would then guide its future use in treatment studies.

\section{Methods}

\section{Sites for data collection}

Data were collected at the University of California, Los Angeles (UCLA) and at two national Dup15q syndrome family conferences. In order to ensure that there were no site differences in EEG outcome, a one-way analysis of variance (ANOVA) with post-hoc tests was performed. There was no evidence of an effect of site on beta power $(F(2,38)=0.28 ; p=0.75)$ or beta peak frequencies $(F(2,38)=1.10 ; p=0.34)$.

\section{Participants}

Children (age $<18$ years) were clinically referred through the Dup15q clinic at UCLA and the Dup15q Alliance. Combining data collected from the three sites, EEG recordings were analyzed from a total of $n=61$ participants. The flowchart in Fig. 1 shows the participant distribution for each parallel study described in this paper and reasons for exclusion from analysis.

\section{Behavioral assessments}

Participants were administered the following measures: (1) The Mullen Scales of Early Learning (MSEL), which assesses general cognition and development [32]. The MSEL yields standard as well as age-equivalent scores that measure receptive and expressive language, visual reception, and gross and fine motor skills. Verbal and non-verbal cognition scores were then calculated. Given that most of our children with Dup15q syndrome had significant delays in overall development, age-equivalent ratio scores were used instead of standardized development quotient scores. (2) Vineland Adaptive Behavior Scale (VABS), a parent reported measure of adaptive behavior, which yields standard and age-equivalent scores for communication, daily living skills (DLS), and socialization and motor skills [33].

\section{EEG data acquisition}

All data were collected under protocols approved by the Institutional Review Board (IRB) (\# 15-001565). Highdensity research EEG data were acquired at a sampling rate of $500 \mathrm{~Hz}$ using 129 channel vertex-referenced Philips Neuro (Eugene, OR, USA) nets with $\mathrm{Ag} / \mathrm{AgCl}$ electrodes. Full details of the research EEG acquisition can be found in a previous publication [4]. For the reproducibility analysis, we accessed overnight clinical EEG recordings that were collected at UCLA as part of routine epilepsy monitoring. These EEGs were collected at a sampling rate of $200 \mathrm{~Hz}$ using a 21-channel 10-20 montage data acquisition set up.

\section{EEG data elimination}

We eliminated data from participants with (1) medications that are known to pharmacologically induce beta oscillations (benzodiazepines or barbiturates), (2) poor data quality due to artifacts from non-neural sources, or (3) lack of measurable peak (i.e., local maximum) in the beta band of the EEG power spectrum. Our final cohort 


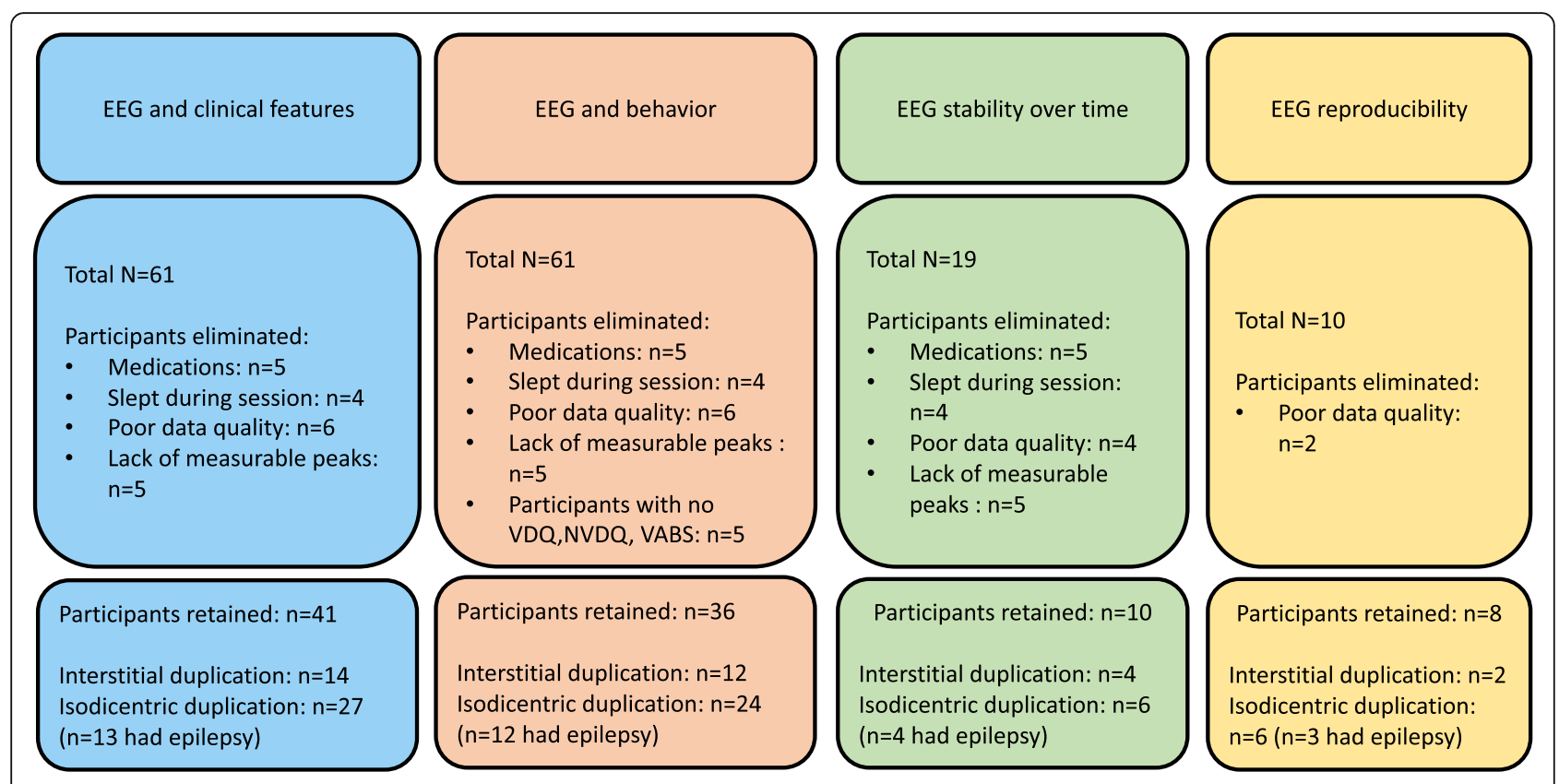

Fig. 1 Flowchart showing participant distribution for each study

of children with Dup15q syndrome yielded $n=41$ participants. Details of age, sex, duplication type, epilepsy status, medications, and IQ can be found in Table 1.

Of the participants that had at least two research EEG recordings from multiple visits, 4 had recordings from three visits. We selected recordings that were at least 10 months apart to enforce this duration of time as a buffer between repeated observations. A total of 10 participants were included in the longitudinal stability analysis. See Table 2 for details of age, epilepsy status (at the time of visit), and duplication type. Dosages of medications were not confirmed with each participant's physician and, therefore, were not included in the table. A subset of our sample $(n=8)$ had additional EEG recorded in a clinical setting for epilepsy monitoring.

\section{EEG Data processing}

Data collection for the described studies spanned several years, and data were processed at two different sites (site 1, Hoffmann-La Roche, Ltd., Basel, Switzerland; site 2, UCLA, Los Angeles, USA) with two different data processing pipelines. To confirm the consistency of the quantified biomarker between different analytic approaches, we compared beta power and peak frequency estimates from data that were processed through both pipelines. Results showed strong reproducibility between analytic pipelines and encouraged us to use the same descriptions of EEG variables ("beta power" and "beta peak frequency") for output from both pipelines despite differences in processing (see Results section for details). Of note, processing methods remained consistent within each study aim (i.e., data from different pipelines were not mixed within one analysis).

For studies 1 and 2 (relation to clinical and behavioral features), EEG data were analyzed at site 1 as an extension of recent work elucidating the mechanism of the EEG biomarker [5] using a combination of in-house tools and the MATLAB software toolbox Fieldtrip [34]. EEG signals were bandpass filtered $1-45 \mathrm{~Hz}$ using a finite impulse response filter (FIR). Sections of data containing gross artifacts and noisy channels were identified by visual inspection and excluded from analysis. Next, noisy channels were marked bad and excluded from subsequent independent component analysis (ICA); a statistical blind source separation technique was implemented to remove physiological artifacts including eye blinks, saccades, ballistocardiogram, and muscle activity, using the Fast ICA algorithm [35, 36]. Finally, rejected channels were spatially interpolated and data were rereferenced to average (in all studies, datasets were discarded when the number of bad channels exceeded the square root of the total number of channels). To derive spectral power estimates, logarithmically scaled frequencies with a spectral smoothing using Morlet wavelets were employed ( 2 to $45 \mathrm{~Hz}, 12$ wavelets per octave) [37]. Power was then averaged across successive 3/4-overlapping temporal windows of continuous clean data after discarding time points corresponding to artifacts. Next, power was normalized with respect to the $\log _{2}$ (frequency), resulting in a power spectral density (PSD) with units of $\mu V 2 / \log 2(\mathrm{~Hz})$ (i.e., power per octave) rather than $\mu \mathrm{V} 2 / \mathrm{Hz}$, thus accounting for the logarithmic nature 
Table 1 Dup15q syndrome participant characteristics (cross-sectional study)

\begin{tabular}{|c|c|c|c|c|c|c|c|}
\hline Age (months) & Gender & Medications (generic) & Duplication Type & Epilepsy (active) & Verbal DQ & Non-verbal DQ & VABS_DLS \\
\hline 9 & M & None & Interstitial & No & 66.67 & 61.11 & 106.00 \\
\hline 18 & M & None & Isodicentric & No & 50.00 & 52.78 & 77.00 \\
\hline 29 & M & Oxcarbazepine & Isodicentric & Yes & 15.52 & 20.69 & 51.00 \\
\hline 38 & M & Levetiracetam & Isodicentric & Yes & 13.16 & 25.00 & 38.00 \\
\hline 39 & M & None & Isodicentric & No & 30.77 & 32.05 & 60.00 \\
\hline 42 & M & None & Isodicentric & No & N/A & N/A & 57.00 \\
\hline 44 & M & None & Interstitial & No & 91.00 & 68.00 & 83.00 \\
\hline 46 & $\mathrm{~F}$ & None & Isodicentric & No & 16.30 & 2.17 & 53.00 \\
\hline 47 & M & None & Isodicentric & No & 73.40 & 69.15 & 83.00 \\
\hline 48 & M & None & Interstitial & No & N/A & N/A & 66.00 \\
\hline 50 & $\mathrm{~F}$ & None & Isodicentric & No & 63.00 & 46.00 & 73.00 \\
\hline 51 & M & None & Isodicentric & No & 32.35 & 38.24 & 54.00 \\
\hline 53 & M & Lamotrigine & Isodicentric & Yes & 9.43 & 19.81 & 57.00 \\
\hline 54 & $\mathrm{~F}$ & None & Interstitial & No & 85.19 & 97.22 & 81.00 \\
\hline 54 & M & None & Interstitial & No & 8.33 & 24.07 & 48.00 \\
\hline 57 & M & None & Isodicentric & No & 47.37 & 44.74 & N/A \\
\hline 61 & $\mathrm{~F}$ & $\begin{array}{l}\text { Valproic acid, clobazam, lacosamide, } \\
\text { perampanel }\end{array}$ & Isodicentric & Yes & 9.02 & 13.93 & 50.00 \\
\hline 62 & $\mathrm{~F}$ & Rufinamide, valproic acid & Isodicentric & No & 50.00 & 30.65 & 57.00 \\
\hline 64 & M & Lamotrigine, rufinamide & Isodicentric & Yes & 10.16 & 15.63 & 45.00 \\
\hline 65 & M & None & Isodicentric & Yes & 9.23 & 13.08 & 38.00 \\
\hline 67 & $\mathrm{~F}$ & None & Interstitial & No & 37.31 & 37.31 & 57.00 \\
\hline 68 & $\mathrm{~F}$ & None & Interstitial & No & 14.71 & 21.32 & N/A \\
\hline 69 & M & Valproic acid, topiramate & Isodicentric & Yes & 12.32 & 13.04 & 45.00 \\
\hline 78 & M & None & Interstitial & No & 19.87 & 21.15 & 50.00 \\
\hline 82 & M & Valproic acid, oxcarbazepine & Isodicentric & Yes & N/A & N/A & 36.00 \\
\hline 94 & M & None & Isodicentric & No & 54.00 & 39.00 & N/A \\
\hline 96 & $\mathrm{~F}$ & None & Isodicentric & No & N/A & N/A & 61.00 \\
\hline 100 & M & Carbamazepine, levetiracetam & Isodicentric & Yes & 54.00 & 64.00 & 66.00 \\
\hline 102 & $\mathrm{~F}$ & None & Isodicentric & No & 34.31 & 22.55 & 58.00 \\
\hline 106 & M & None & Interstitial & No & N/A & $\mathrm{N} / \mathrm{A}$ & 71.00 \\
\hline 108 & M & None & Interstitial & No & 64.35 & 60.65 & 73.00 \\
\hline 111 & $\mathrm{~F}$ & None & Interstitial & No & 26.58 & 23.87 & 55.00 \\
\hline 118 & $\mathrm{~F}$ & None & Interstitial & No & 36.86 & 44.07 & N/A \\
\hline 127 & $\mathrm{~F}$ & Topiramate & Isodicentric & Yes & 22.83 & 20.47 & 47.00 \\
\hline 134 & M & None & Isodicentric & No & 9.70 & 18.66 & 38.00 \\
\hline 153 & M & $\begin{array}{l}\text { Rufinamide, levetiracetam, lacosamide, } \\
\text { epidolex }\end{array}$ & Isodicentric & Yes & 24.18 & 22.22 & N/A \\
\hline 156 & M & None & Isodicentric & No & 37.18 & 24.20 & 40.00 \\
\hline 161 & M & None & Interstitial & No & 73.00 & 66.00 & 73.00 \\
\hline 169 & M & Rufinamide, valproic acid & Isodicentric & Yes & 16.57 & 13.02 & 20.00 \\
\hline 175 & $\mathrm{~F}$ & None & Interstitial & No & 31.00 & 41.00 & 86.00 \\
\hline 189 & M & None & Isodicentric & Yes & 40.00 & 36.00 & 82.00 \\
\hline
\end{tabular}

Cognitive tests were not available for all the participants. N/A not available. Dosages were not available for all the medications listed, hence not included in the table 
Table 2 Dup15q syndrome participant characteristics (longitudinal study)

\begin{tabular}{|c|c|c|c|c|c|}
\hline Participant & $\begin{array}{l}\text { Duplication } \\
\text { type }\end{array}$ & $\begin{array}{l}\text { Visit } 1 \text { Age } \\
\text { (months) }\end{array}$ & $\begin{array}{l}\text { Visit } 2 \text { Age } \\
\text { (months) }\end{array}$ & Epilepsy (active) & Medications \\
\hline P1 & Isodicentric & 57 & 72 & No & N/A \\
\hline P2 & Isodicentric & 18 & 28 & $\begin{array}{l}\text { Yes (at second } \\
\text { visit) }\end{array}$ & Vigabatrin (at second visit) \\
\hline P3 & Interstitial & 51 & 78 & No & N/A \\
\hline P4 & Isodicentric & 110 & 134 & No & N/A \\
\hline P5 & Isodicentric & 84 & 100 & Yes (at both visits) & $\begin{array}{l}\text { Carbamazepine; levetiracetam (unchanged at both } \\
\text { visits) }\end{array}$ \\
\hline P6 & Interstitial & 48 & 72 & No & N/A \\
\hline P7 & Isodicentric & 29 & 53 & $\begin{array}{l}\text { Yes (at second } \\
\text { visit) }\end{array}$ & Lamotrigine (at second visit) \\
\hline P8 & Isodicentric & 38 & 61 & Yes (at both visits) & $\begin{array}{l}\text { Valproate, clobazam, lacosamide, perampanel } \\
\text { (unchanged at both visits) }\end{array}$ \\
\hline P9 & Interstitial & 44 & 68 & No & $\mathrm{N} / \mathrm{A}$ \\
\hline P10 & Interstitial & 161 & 185 & No & $\mathrm{N} / \mathrm{A}$ \\
\hline
\end{tabular}

N/A not available. Dosages were not available for all the medications listed, hence not included in the table

of electrophysiological signals [38]. We reported beta power using trapezoidal integration in the $12-30 \mathrm{~Hz}$ band (MATLAB: trapz, absolute power integrated with respect to $\log _{2}$ (frequency)). For further details of data processing see Frohlich et al. [5]

For studies 3 and 4, a separate data processing pipeline was applied using the EEGLAB software toolbox [39] for MATLAB. In this pipeline, data were FIR filtered 1-45 Hz. Sections of data containing gross artifacts and noisy channels were identified by visual inspection and excluded from analysis. Data were interpolated to a 25channel montage before using ICA (infomax algorithm; EEGLAB: "runica") to remove physiological artifacts. Data were then re-referenced to an average of all channels. For each electrode, PSDs were computed according to Welch's method [4, 40], with power normalized per $\mathrm{Hz}$ (yielding $\mu \mathrm{V} 2 / \mathrm{Hz}$ ). Beta power was reported as the sum of the absolute power in the 12$30 \mathrm{~Hz}$ band.

In order to extract peak frequencies within the beta band, power spectra computed using aforementioned methods were averaged across electrodes. Peak labeling was performed automatically using the local maximum in the beta band, as well as manually using visual inspection. Manual labeling was performed by two trained raters. Raters were blinded to epilepsy status. In instances of manual labeling, an average of the peak labeling values obtained from the two raters was used. For each participant, automatically and manually labeled peak frequencies were compared. When the automated peak labeling fell within $5 \%$ of the value of the manual peak labeling, values from automated labeling were used. Otherwise, values from manual labeling were used.

\section{Data analysis}

In order to ensure that there were no differences in the dependent variables between the two data processing methods, we compared recordings from participants $(n$ $=8$ ) that were processed using both pipelines, and intraclass correlation coefficients (ICC) were derived.

Studies 1 and 2: Relation to clinical and behavior features To determine the relation between EEG and clinical features, simple linear regression models were performed using beta power and BPF as outcome measures, and duplication type, epilepsy status, and age as separate predictors of beta power and BPF. Age was treated as a continuous variable, while duplication type and epilepsy were treated as binary variables. Next, to determine the relationship between EEG and behavior, beta power and peak frequency were regressed on quantitative measures of cognition, as well as parent reported measures of social skills.

\section{Studies 3 and 4: EEG stability and reproducibility}

To evaluate stability of spectral power and peak frequency in the beta band across time points, ICCs were derived from EEG recordings of all participants with more than one research visit. To evaluate reproducibility of the EEG signature in Dup15q syndrome, beta power was compared between (a) high-density research EEG recorded from participants while they were awake and resting, and (b) low-density clinical EEG collected for epilepsy monitoring, with data extracted from segments in which participants were awake and resting prior to entering sleep. Data from low-density clinical EEG and high-density research EEG was computed and compared through derived ICCs. 
Because our studies were performed in parallel, with no primary analysis selected between the four, we did not correct for multiple comparisons within or across the studies. Ninety-five percent confidence intervals (CIs) and effect sizes (where applicable) are reported to allow the reader to better interpret the meaningfulness of each finding.

\section{Results}

The comparison of the two different pre-processing pipelines (site 1 and site 2) yielded an ICC of 0.93 (95\% confidence interval 0.67-0.99) for beta power and an ICC of 0.92 (95\% confidence interval 0.64-0.98) for BPF, indicating moderate to excellent correlation between the two data processing methods.

\section{Study 1: Relation to clinical features (age, duplication} type, and epilepsy)

Neither beta power $\left(R^{2}=0.014,95 \% \mathrm{CI}-0.41\right.$ to $0.19, p$ $=0.47)$ nor $\mathrm{BPF}\left(R^{2}=0.005,95 \% \mathrm{CI}-0.24\right.$ to $0.37, p=$ $0.65)$ correlated with age (Fig. 2a, b). There were no differences in beta power between duplication types (Fig. 3a; $R^{2}=5 \times 10^{-5}, 95 \% \mathrm{CI}-0.31$ to $0.30, p=$ 0.96). BPF did not differ between duplication types. Individual beta band peaks derived from participants in the two duplication groups are shown in Fig. 3b, and the average peak frequency for the isodicentric and interstitial duplication groups were $22.6 \mathrm{~Hz}$ and $23.1 \mathrm{~Hz}$, respectively. Mean topographic distribution of power across the scalp at the mean peak frequency is shown for each duplication type (interstitial, Fig. 3c, isodicentric, Fig. 3d). Both duplication types showed excessive beta power similar to that found in previous work $[4,5]$.

Beta power did not significantly differ between those with and without epilepsy $\left(R^{2}=3 \times 10^{-4}, 95 \% \mathrm{CI}-0.28\right.$ to $0.32, p=0.90$, Fig. 4a). However, BPF did significantly differentiate groups, with children with epilepsy showing a significantly lower peak frequency compared to those without epilepsy (correlation: $R^{2}=0.11,95 \%$ CI: -0.58 to $-0.02, p=0.038$; $t$ test: $t=2.15,95 \%$ CI $0.08-2.86, d=$ 0.07). Individual peaks captured for each participant in the beta frequency range are shown in Fig. 4b, with average peak frequency within the epilepsy and non-epilepsy groups being $21.8 \mathrm{~Hz}$ and $23.3 \mathrm{~Hz}$, respectively. Mean topographic distribution of power across the scalp at the mean group-level peak frequency is shown for epilepsy and non-epilepsy groups in Fig. $4 \mathrm{c}$ and d.

\section{Study 2: Relation to cognition and adaptive skills}

Behavioral testing is summarized in Table 1. Regression models to investigate predictors of beta power revealed that verbal and non-verbal cognition, and DLS, were not predictors of beta power (VDQ: $R^{2}=$ 0.005, $p=0.68$, NVDQ: $R^{2}=0.0002, p=0.94$, DLS: $\left.R^{2}=0.0002, p=0.97\right)$.

We also performed regression models within the epilepsy and non-epilepsy groups separately and did not find meaningful associations (non-epilepsy group: VDQ: $R^{2}=0.005,95 \% \mathrm{CI}-0.34$ to $0.46, p=0.75$, NVDQ: $R^{2}=$ $0.0002,95 \%$ CI -0.42 to $0.39, p=0.94$, DLS: $R^{2}=0.024$, $95 \% \mathrm{CI}-0.53$ to $0.26, p=0.46$; epilepsy group: VDQ: $R^{2}$ $=0.17,95 \% \mathrm{CI}-0.21$ to $0.80, p=0.18$, NVDQ: $R^{2}=$

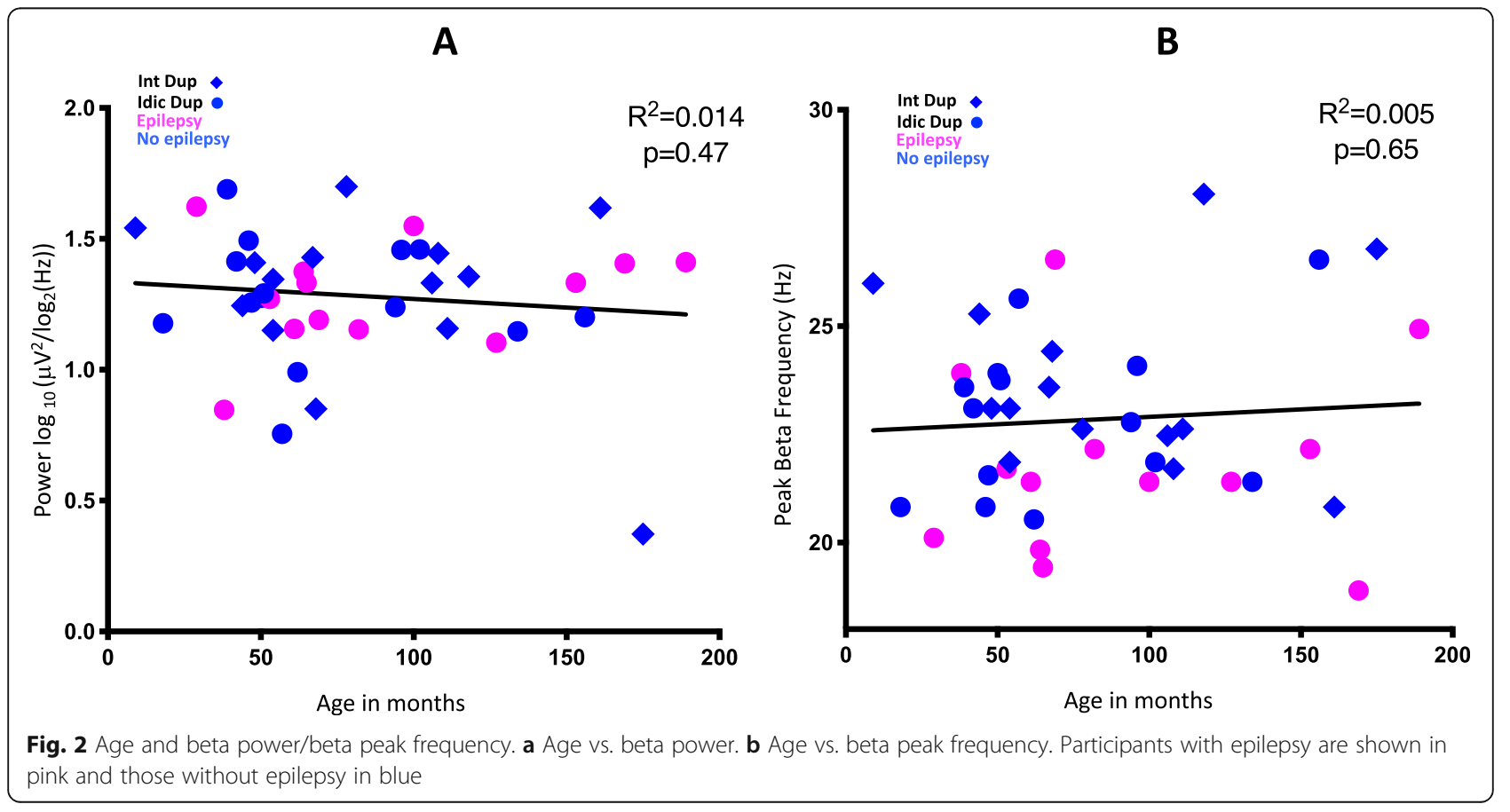




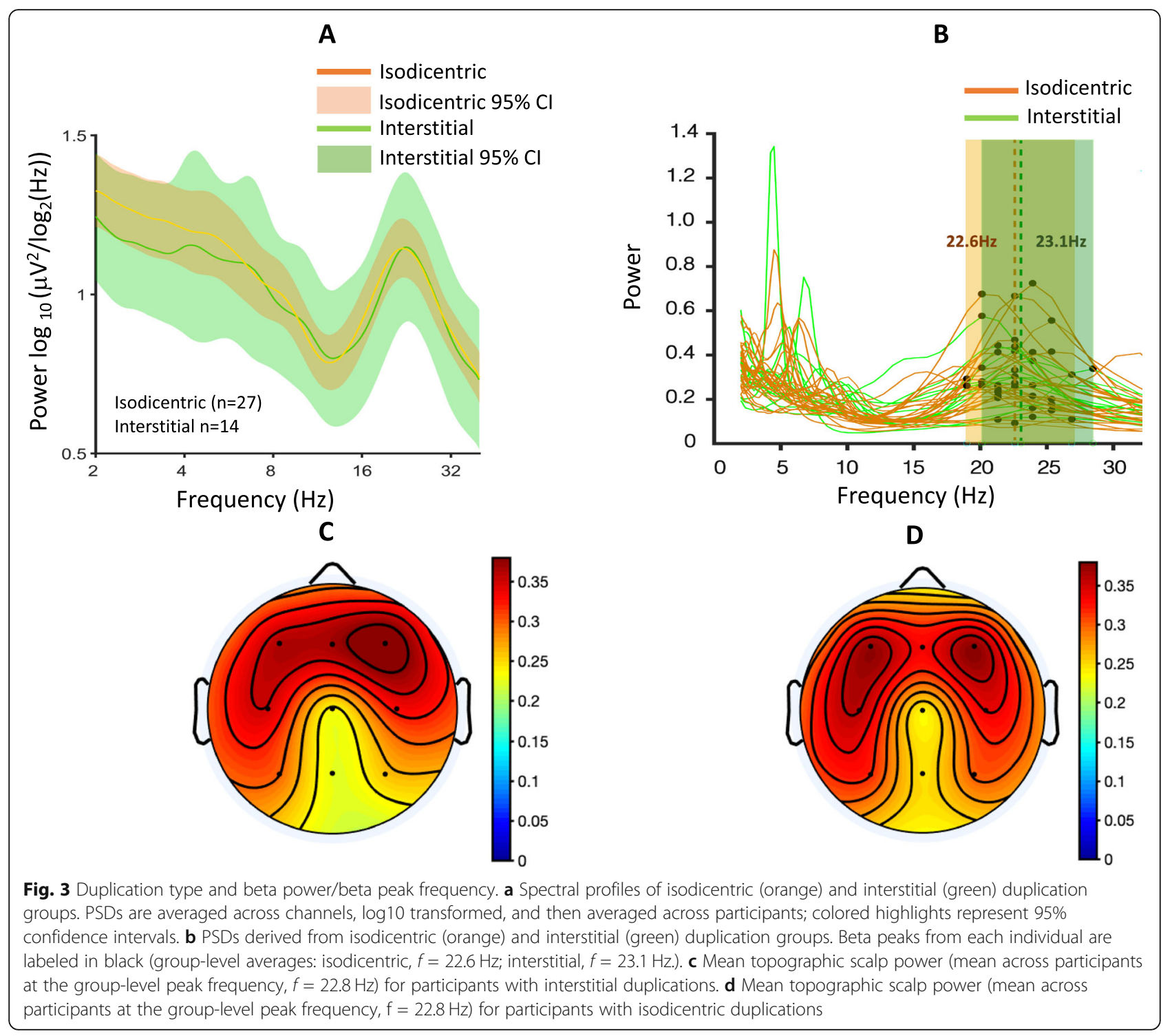

$0.11,95 \%$ CI -0.30 to $0.76, p=0.29$, DLS: $R^{2}=0.11$, 95\% CI -0.30 to $0.76, p=0.30$ ) (Fig. $5 \mathrm{a}-\mathrm{c}$ ).

Neither verbal nor non-verbal cognition predicted BPF within the Dup15q syndrome cohort (VDQ: $R^{2}=0.049$, $p=0.19$, NVDQ: $R^{2}=0.055, p=0.17$ ). Upon performing regression models within the epilepsy and non-epilepsy groups separately, we found no significant relationship within the non-epilepsy group (non-epilepsy group: VDQ: $R^{2}=0.002,95 \%$ CI -0.36 to $0.44, p=0.82$, NVDQ: $R^{2}=0.012,95 \% \mathrm{CI}-0.31$ to $0.49, p=0.61$; epilepsy group: VDQ: $R^{2}=0.035,95 \% \mathrm{CI}-0.43$ to $0.69, p$ $=0.56$, NVDQ: $R^{2}=0.024,95 \%$ CI -0.46 to $0.67, p=$ 0.63) (Fig. 6a, b). A moderate correlation between BPF and measure of DLS was seen, in that participants with lower adaptive skills had significantly lower peak frequency $\left(R^{2}=0.16695 \%\right.$ CI $\left.0.09-0.65, p=0.01\right)$. This relationship may be driven by individuals who have epilepsy, as participants with epilepsy have lower DLS scores and lower BPF [epilepsy group: mean DLS score $=47.9$, mean $\mathrm{BPF}=21.8 \mathrm{~Hz}]$ compared to those in the non-epilepsy group [non-epilepsy: mean DLS score = 65.0, mean $\mathrm{BPF}=23.0 \mathrm{~Hz}]$. Nonetheless, $\mathrm{BPF}$ accounted for a similar proportion of DLS variance in the epilepsy subgroup as in the overall cohort (epilepsy group: $R^{2}=$ $0.17,95 \% \mathrm{CI}-0.21$ to $0.79, p=0.18$; non-epilepsy group: $R^{2}=0.08,95 \%$ CI -0.13 to $0.61, p=0.19$ ) (Fig. 6c), suggesting a possible correlation within the epilepsy subgroup that we were underpowered to detect.

\section{Study 3: Stability over time}

The ICC derived from beta power from participants that had at least two EEGs was 0.93 (95\% CI 0.63-0.98). ICC derived from BPFs from the same participants was 0.92 (95\% CI 0.64-0.98), indicating moderate to excellent stability of both 

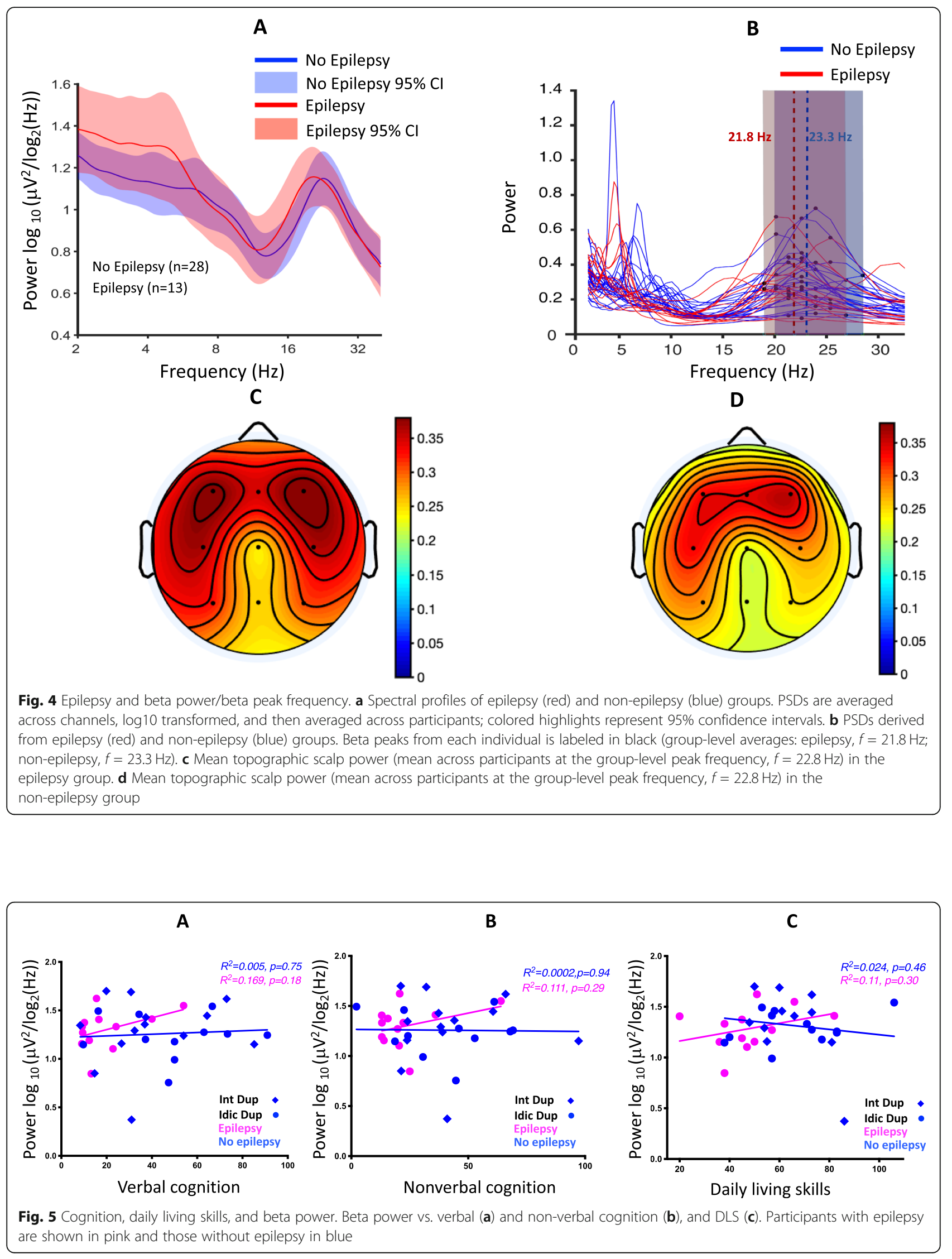
A

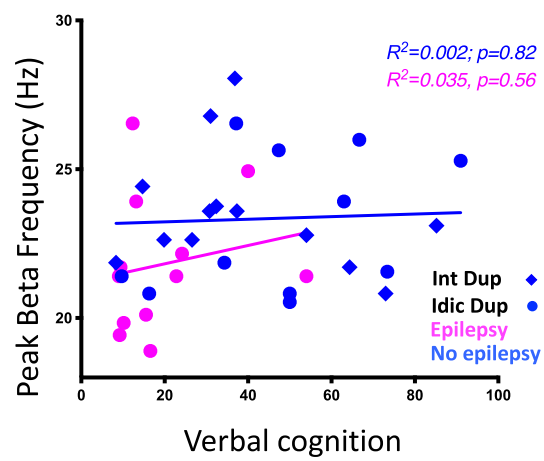

B

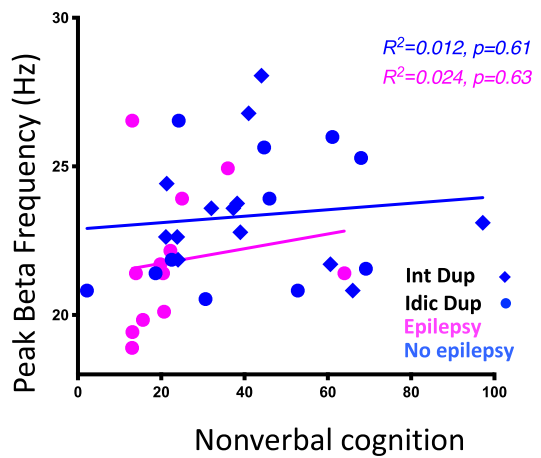

C

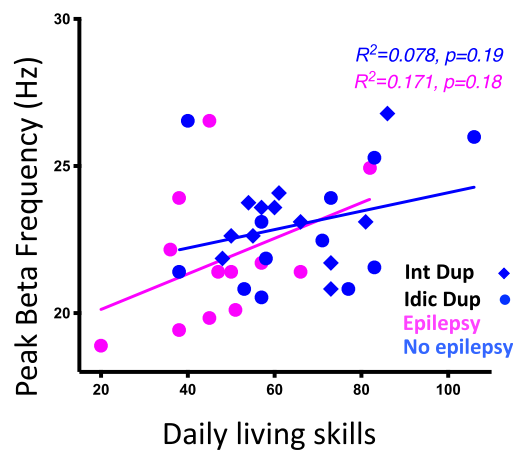

Fig. 6 Cognition, daily living skills, and beta peak frequency. a, b Beta peak frequency vs. verbal and non-verbal cognition. c Beta peak frequency vs. DLS. Participants with epilepsy are shown in pink and those without epilepsy in blue

beta power and BPF across multiple EEG recordings (Fig. 7). Since two out of the ten participants developed epilepsy between visits, they were excluded from the ICC analysis.

\section{Study 4: Reproducibility from research to clinical EEG}

The ICC derived from spectral power values computed from participants with high-density research and lowdensity clinical EEG recordings was 0.94 (95\% confidence interval 0.60-0.98), indicating moderate to excellent reproducibility of the biomarker from research to clinical recordings. Figure 8 shows PSD plots of research EEGs (top row) and clinical EEGs (bottom row) of two representative participants.

\section{Discussion}

Elevated beta band oscillations represent a robust, easily measurable biomarker of Dup15q syndrome, a genetic variant highly penetrant for NDDs and a promising target for future clinical trials, particularly disease modifying therapies. Here, we extended our quantification of this biomarker by testing properties that would inform its use in future trials, namely its relation to clinical and behavioral features, stability over time, and reproducibility across data collection systems and analytic pipelines. Key results included (1) differences in BPF between those with and without epilepsy, (2) stability over time based on consistency of signal between two EEG recordings at least 10 months apart, (3) reproducibility between

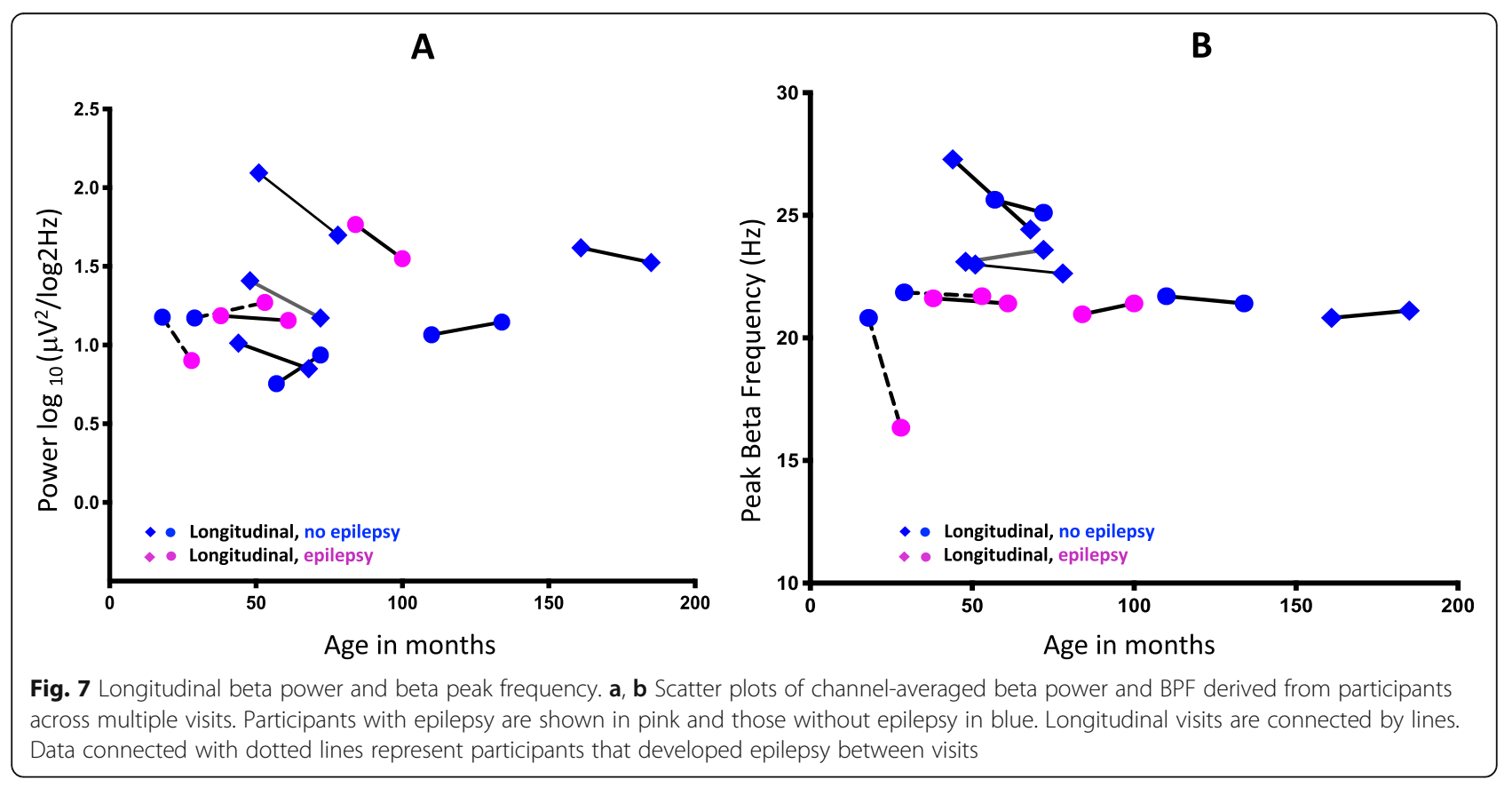




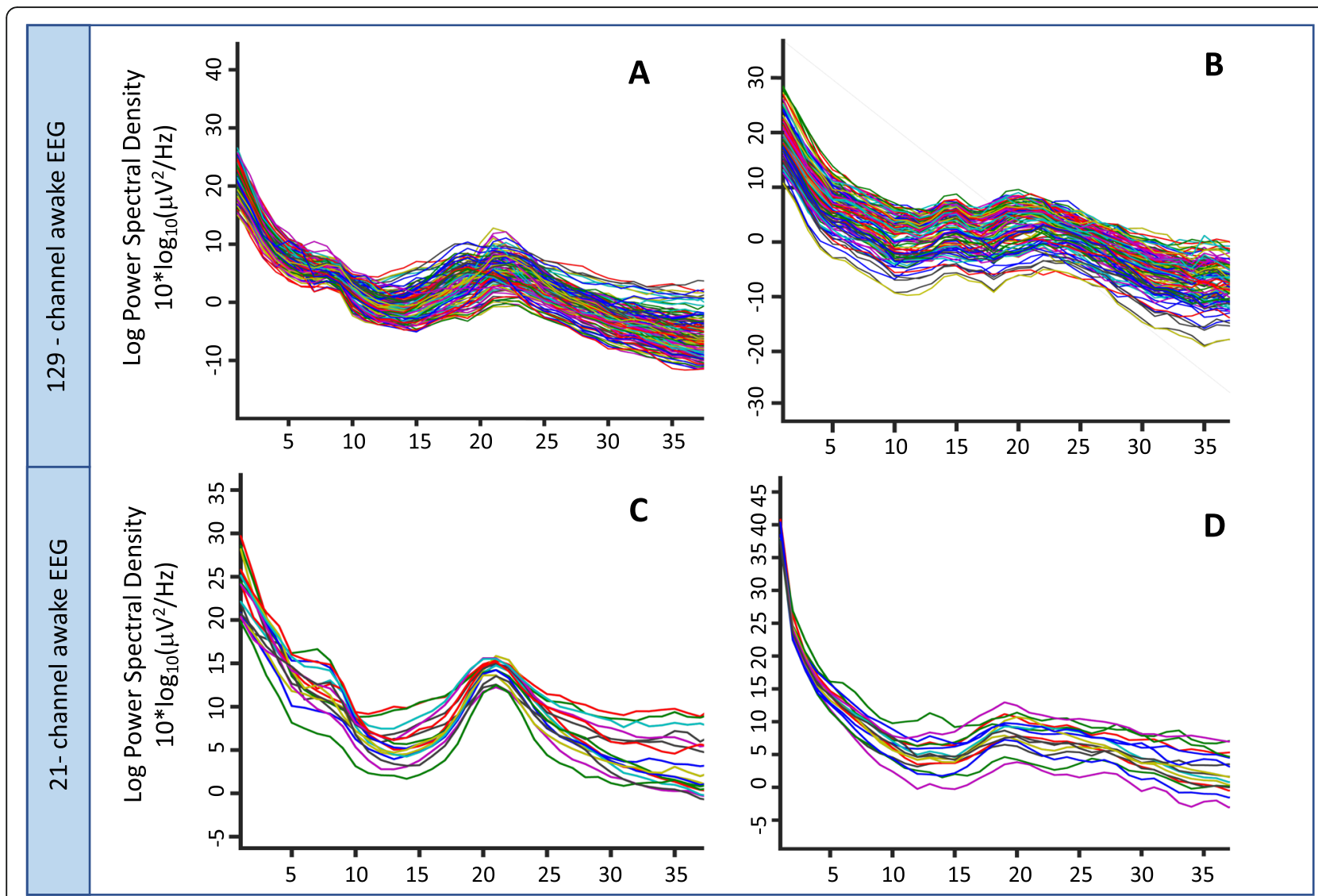

Fig. 8 High- vs. low-density EEG PSDs. PSDs derived from research EEG (top row) and clinical EEG (bottom row) from two participants are shown. a, $\mathbf{c}$ PSDs from research and clinical EEG respectively of first participant. $\mathbf{b}, \mathbf{d}$ PSDs from research and clinical EEG respectively of second participant. Individual channels are shown in different colors

research and clinical EEG recordings obtained as part of children's routine epilepsy monitoring, and (4) reproducibility across two analytic pipelines that employed different frequency transforms and normalization.

\section{Beta oscillations in Dup15q syndrome}

Spontaneous beta oscillations typically observed in human EEG reflect a state of cortical activation [41] through a network of inhibitory interneurons and pyramidal cells [42]. The period (and thus frequency) of neural oscillations is determined in part by the time constants on postsynaptic receptors (i.e., faster time constants yields faster oscillatory frequencies), as is known to be true of gamma oscillations and $\mathrm{GABA}_{\mathrm{A}} \mathrm{Rs}$ [43]. To this effect, barbiturates such as phenobarbital and pentobarbital increase the duration of $\mathrm{GABA}_{\mathrm{A}} \mathrm{R}$ channel opening $[44,45]$, as does zolpidem, a benzodiazepine-like compound (described as a "benzodiazepine agonist" in older literature) [46, 47]. Moreover, benzodiazepines decrease the frequency of beta oscillations [42, 47], lending plausibility to the idea that increases in beta power observed with both pharmacological $\mathrm{GABA}_{\mathrm{A}} \mathrm{R}$ modulation and $15 \mathrm{q}$ duplication result from shifting of fast oscillations towards a resonate frequency in beta (i.e., the frequency the system prefers to oscillate at when energy is added), thus explaining the very large amplitude of beta seen in both contexts. This mechanism is speculative, and it remains unknown how it would interact with other factors (e.g., epilepsy and receptor properties altered by antiepileptic medication). Nevertheless, based on the hypothesized role of $\mathrm{GABA}_{\mathrm{A}} \mathrm{Rs}$ in the generation of these oscillations, the EEG biomarker in Dup15q syndrome could enrich clinical trials by serving as a measure of drug target engagement or as a proximal outcome measure that precedes behavioral responses to pharmacological treatments that modulate GABAergic neurotransmission.

\section{Beta oscillations and relationship to phenotype}

We found no significant relationship between beta parameters and age, demonstrating that beta power and frequency are likely readouts of the fundamental disease pathology in Dup15q syndrome that remains unchanged over development. There is increased interest in 
identifying biomarkers of NDDs that relate to clinical symptomatology. We found that the strongest clinical predictor of the EEG signature in Dup15q syndrome was epilepsy, as BPF differed based on epilepsy status.

As in many syndromic NDDs, epilepsy in Dup15q syndrome is associated with greater functional impairment [48]. We urge caution in not overinterpreting the relationship between epilepsy and beta oscillations, as without preclinical models to manipulate the underlying altered circuitry, we will not be able to prove directionality of the association. Epilepsy (both active seizures and interictal epileptiform EEG activity) causes significant changes in the EEG, such as slowing of background oscillations and spike/wave discharges, and these changes have been demonstrated in overnight EEG studies of patients with Dup15q syndrome [49]. Antiepileptic medications also change oscillatory activity, with some medications, such as $\mathrm{GABA}_{\mathrm{A}} \mathrm{R}$ positive allosteric modulators, causing further elevation of high frequency oscillations, while others, such as phenytoin or carbamazepine, increasing generalized cortical slowing. If and how seizures and/or their treatments slow the $\mathrm{GABA}_{\mathrm{A}} \mathrm{R}$ time constant to produce a slower beta frequency is not fully understood. This question may be best addressed in preclinical models, in which various aspects of the circuits can be directly manipulated. However, from a clinical perspective, this association has tremendous promise, and future studies will assess whether BPF serves as a predictor of epilepsy early in development or as an informative marker of response to antiepileptic drug studies.

We found no correlation between duplication type and beta power or BPF. This negative result should be interpreted with caution: The number of individuals with EEG and clinical data is limited, which enforced (1) combining different clinical assessments of cognition (MSEL, Differential Ability Scales), (2) combining a broad age range, (3) combining both genotypes (interstitial, isodicentric), and (4) combining individuals with and without epilepsy. It is possible that future analyses, possibly with more participants, that account for those sources of variance will find a relation. However, it is also well possible that a relation between EEG Beta oscillations does not exist. Beta oscillations are an emergent network property that may nonlinearly increase and then asymptomatically saturate based on $\mathrm{GABA}_{\mathrm{A}} \mathrm{R}$ expression. In Dup15q syndrome (both interstitial and isodicentric), such a saturation may be reached (compatible with finding no higher beta in isodicentric duplications despite a presumably higher genedose effect). Therefore, there may not be room to measure inter-subject variability that could correlate with symptom severity. Thus, even in the absence of a correlation with symptom severity, elevated beta oscillations in Dup15q syndrome could still be a useful biomarker of altered GABAergic neurotransmission that could be used in drug development. The saturation of beta oscillations based on $\mathrm{GABA}_{\mathrm{A}} \mathrm{R}$ expression could, for instance, be explained biophysically by the short duration of the beta cycle (33-83 $\mathrm{ms})$, which limits the number of neurons that can be recruited for the oscillation [50]. Future studies with computational models backed by experiments will be needed to assess changes of individual cellular biophysical parameters to network oscillation.

We found no correlation between behavior and beta power or BPF in Dup15q syndrome. Although BPF was associated with adaptive skills, this relationship may be driven by epilepsy status, as children with epilepsy have lower overall adaptive skills compared to those without epilepsy [51-54]. It is possible that our behavioral measures lack sufficient sensitivity to capture the range of clinical variability in individuals with Dup15q syndrome. However, our results build on findings in Angelman syndrome showing that children with 15q11-q13 deletions (deletion Angelman) have lower beta $(23 \mathrm{~Hz})$ power than children with other etiologies that principally affect UBE3A (nondeletion Angelman) [24]. In fact, deletion and nondeletion Angelman differ not only in beta power but also in clinical severity [55-58]. Given that beta power and clinical severity covary in Angelman, it is possible that a similar relationship exists in Dup15q syndrome, but that either the severity of cognitive impairment in this population or other limitations to the psychometric properties of the tests used in this sample may limit our ability to capture subtle relationships between this biomarker and behavior. However, this lack of correlation with behavior does not undermine its potential for use as a marker of drug target engagement in clinical trials, as here we did not test whether change in beta oscillations predict or relate to change in clinical outcomes.

\section{Stability and reproducibility of the EEG biomarker}

Our data show stability across multiple recordings and reproducibility across data acquisition (research vs clinical EEG) methods. A fundamental question addressed by our study was whether this biomarker could be quantified from low-density clinical recordings performed outside of research study setting, which is of particular importance given that most of these children undergo clinical EEGs regularly as part of their clinical monitoring. One of the biggest challenges in EEG studies in NDDs is data collection itself (i.e., bringing in participants to sites to collect the research EEG). Future efforts that would bypass data collection in an expensive, structured research setting and quantify beta power and frequency in a repository of clinical EEGs would allow additional analyses to be far more clinically relevant, scalable, and statistically powered. In fact, our study has already motivated these analyses in an ongoing clinical 
trial for epilepsy in Dup15q syndrome, with beta power being quantified through clinical EEG collected at baseline.

\section{Biomarkers in neurodevelopmental disorders}

Research in preclinical models has truly advanced our insights into the pathological mechanisms underlying various NDDs. Despite this knowledge, even presumably well-designed clinical trials have struggled to demonstrate significant effects in treatment groups compared to placebo $[59,60]$. These challenges may reflect several gaps: (1) even when the treatment has a clear biological, mechanistic target, lack of measurement of drug target engagement limits the ability to determine if a drug could have an effect; (2) in the setting of the biological and developmental heterogeneity of these conditions, there are few objective methods for patient selection for trials; (3) outcome measures themselves often prove ineffective to capture the effect of a treatment because of insensitivity to short term change or vulnerability to reporting bias or placebo effect; and (4) outcome measures chosen based on preclinical research do not translate to meaningful or modifiable patient-centered outcomes. The identification and quantification of objective biomarkers can mitigate some of these challenges by facilitating patient stratification, measuring drug target engagement, and defining outcomes relatively resistant to the placebo effect.

As EEG can measure circuit-level treatment response before behavior changes can be observed, EEG biomarkers have the potential to address the challenges involved in pharmacological trials [60]. To that end, this study holds promise in identifying EEG biomarkers in a rare genetic population highly penetrant for NDDs. The lack of a detectable association between the EEG biomarker and age or symptom severity in this sample demonstrates that this biomarker likely relates to the underlying neurobiology in Dup15q syndrome, with possible relation to GABAergic dysfunction. The hypothesis that we can quantitatively measure the impact of altered GABA signaling is particularly exciting in the field of NDDs, as dysfunction in the dynamics of cortical GABAergic circuitry may be implicated in syndromes other than Dup15q syndrome. Furthermore, converging evidence from gene linkage studies suggest that point mutations in the $\mathrm{GABA}_{\mathrm{A}} \beta 3 / \alpha 5 / \gamma 3$ gene cluster may also be implicated in other NDDs $[7,61,62]$. It is possible that elevated beta oscillations in individuals with other NDDs may herald other genetic causes of altered GABA neurotransmission, such as point mutations in the $\mathrm{GABA}_{\mathrm{A}} \beta 3 / \alpha 5 / \gamma 3$ gene cluster. Future studies can therefore leverage existing electrophysiological data from children with NDDs and explore the utility of the EEG biomarker in Dup15q syndrome to predict genetic variants in children with NDDs and further our understanding of underlying circuit-level pathology in a subset of these children.

\section{Limitations, conclusions, and future directions}

Our work herein established the robustness and reproducibility of the EEG beta phenotype as a biomarker of Dup15q syndrome. Studies of rare genetic disorders are often limited by small sample number, and we also faced these sample size challenges, particularly in the studies of stability over time and reproducibility. Findings from our reproducibility study have led to the development of a new data acquisition and storage pipeline for clinical overnight sleep EEG recordings of children with Dup15q syndrome across the world, in partnership with the Dup15q Alliance. We will utilize these recordings to investigate the presence of beta oscillations in sleep EEG and to characterize sleep physiology in children with the syndrome. As more children with syndromic forms of NDDs undergo clinical EEG investigation, this pipeline will directly guide decisions to replace research EEG recordings with clinical ones, thereby facilitating larger scale studies of EEG biomarkers across syndromic NDDs.

\section{Abbreviations}

Dup15q syndrome: Duplication 15q11.2-q13.1 syndrome; NDD: Neurodevelopmental disorder; EEG: Electroencephalogram; ASD: Autism spectrum disorder; GABA $A_{A}$ : Gamma-aminobutyric acid type A; ID: Intellectual disability; UCLA: University of California, Los Angeles; DQ: Developmental quotient; VABS: Vineland Adaptive Behavior Scale; DLS: Daily living skills; BPF: Beta peak frequency; IRB: Institutional review board; ICA: Independent component analysis; ICC: Intraclass correlation coefficient; PSD: Power spectral density

\section{Acknowledgements}

We warmly thank all the children and families who participated in this research and made this work possible.

\section{Authors' contributions}

VS and JF processed data from children. VS, AWS, and DS analyzed the data. VS produced the figures. JFH and JF wrote the code for data processing in study 1 and 2. VS wrote the manuscript. VS and SSJ planned and conceptualized the study. VS and CH collected data from children. VS, JF, PG, EHC, LTR, DS, and SSJ contributed to the interpretation of the results. SSJ supervised the study. All authors read and approved the final manuscript.

\section{Funding}

This work was supported by the Dup15q Alliance \#20182241

(Saravanapandian), the National Institute of Mental Health of the National Institute of Health T32MH073526 (Saravanapandian), the National Institute of General Medical Sciences \#R01 GM111378-01A1 (Senturk), and the UCLA Intellectual and Developmental Disabilities Research Center U54HD087101-04 (Jeste).

\section{Availability of data and materials}

EEG data from Dup15q syndrome children are available from the corresponding author on reasonable request.

Ethics approval and consent to participate

All EEG studies and analyses were performed with the institutional review board (IRB) approval. 


\section{Competing interests}

Vidya Saravanapandian: no competing interests

Joel Frohlich is a former employee of F. Hoffmann-La Roche Ltd. (October 2016-July 2017).

Joerg F. Hipp is an employee of F. Hoffmann-La Roche Ltd.

Carly Hyde: no competing interests

Aaron W. Scheffler: no competing interests

Peyman Golshani: no competing interests

Edwin H. Cook: no competing interests

Lawrence T. Reiter: no competing interests

Damla Senturk: no competing interests

Shafali Jeste serves as a consultant for and has received funding from F.

Hoffmann-La Roche Ltd. and Yamo Pharmaceuticals

\section{Author details}

${ }^{1}$ Center for Autism Research and Treatment, Semel Institute for Neuroscience, University of California Los Angeles, Los Angeles, CA 90024, USA. ${ }^{2}$ Roche Pharma Research and Early Development, Neuroscience, Ophthalmology and Rare Diseases, Roche Innovation Center Basel, Basel, Switzerland. ${ }^{3}$ Department of Psychology, University of California Los Angeles, 3423 Franz Hall, Los Angeles, CA 90095, USA. ${ }^{4}$ Department of Biostatistics, University of California Los Angeles School of Public Health, Room 21-254C CHS, Los Angeles, CA 90095, USA. ${ }^{5}$ Department of Neurology and Semel Institute for Neuroscience, David Geffen School of Medicine, 710 Westwood Plaza, Los Angeles, CA 90095, USA. ${ }^{6}$ West Los Angeles VA Medical Center, 11301 Wilshire Blvd, Los Angeles, CA 90073, USA. ${ }^{7}$ Department of Psychiatry, University of Illinois at Chicago, 1747 W Roosevelt Road, Chicago, IL 60608, USA. ${ }^{8}$ Department of Neurology, Pediatrics and Anatomy \& Neurobiology, The University of Tennessee Health Science Center, 855 Monroe Ave., Link, Memphis, TN 415, USA.

Received: 17 September 2019 Accepted: 30 July 2020

Published online: 13 August 2020

\section{References}

1. de la Torre-Ubieta $L$, et al. Advancing the understanding of autism disease mechanisms through genetics. Nat Med. 2016;22(4):345-61

2. Berry-Kravis $E$, et al. Outcome measures for clinical trials in fragile $X$ syndrome. J Dev Behav Pediatr. 2013;34(7):508-22.

3. Ferlini A, Scotton C, Novelli G. Biomarkers in rare diseases. Public Health Genomics. 2013;16(6):313-21.

4. Frohlich J, et al. A quantitative electrophysiological biomarker of duplication 15q11.2-q13.1 Syndrome. PLoS One. 2016;11(12):e0167179.

5. Frohlich J, et al. Mechanisms underlying the EEG biomarkessr in Dup15q syndrome. Mol Autism. 2019;10:29.

6. Urraca $\mathrm{N}$, et al. The interstitial duplication 15q11.2-q13 syndrome includes autism, mild facial anomalies and a characteristic EEG signature. Autism Res. 2013;6(4):268-79.

7. Cook EH Jr, et al. Autism or atypical autism in maternally but not paternally derived proximal 15q duplication. Am J Hum Genet. 1997;60(4):928-34.

8. Moreno-De-Luca D, et al. Using large clinical data sets to infer pathogenicity for rare copy number variants in autism cohorts. Mol Psychiatry. 2013;18(10):1090-5.

9. DiStefano $\mathrm{C}$, et al. Identification of a distinct developmental and behavioral profile in children with Dup15q syndrome. J Neurodev Disord. 2016:8:19.

10. Finucane, B.M., et al., 15q duplication syndrome and related disorders, in GeneReviews((R)), M.P. Adam, et al., Editors. 2016, University of Washington, Seattle. University of Washington, Seattle. GeneReviews is a registered trademark of the University of Washington, Seattle. All rights reserved. Seattle (WA).

11. Abrahams BS, Geschwind DH. Advances in autism genetics: on the threshold of a new neurobiology. Nat Rev Genet. 2008;9(5):341-55.

12. Yamasaki $\mathrm{K}$, et al. Neurons but not glial cells show reciprocal imprinting of sense and antisense transcripts of Ube3a. Hum Mol Genet. 2003;12(8):837-47.

13. Dindot SV, et al. The Angelman syndrome ubiquitin ligase localizes to the synapse and nucleus, and maternal deficiency results in abnormal dendritic spine morphology. Hum Mol Genet. 2008;17(1):111-8.

14. Albrecht $U$, et al. Imprinted expression of the murine Angelman syndrome gene, Ube3a, in hippocampal and Purkinje neurons. Nat Genet. 1997;17(1):75-8.
15. Copping NA, et al. Neuronal overexpression of Ube3a isoform 2 causes behavioral impairments and neuroanatomical pathology relevant to $15 q 11$. 2-q13.3 duplication syndrome. Hum Mol Genet. 2017;26(20):3995-4010.

16. Germain ND, et al. Gene expression analysis of human induced pluripotent stem cell-derived neurons carrying copy number variants of chromosome 15q11-q13.1. Mol Autism. 2014;5:44.

17. Al Ageeli $E$, et al. Duplication of the 15q11-q13 region: clinical and genetic study of 30 new cases. Eur J Med Genet. 2014;57(1):5-14.

18. Christian EP, et al. EEG-beta/gamma spectral power elevation in rat: a translatable biomarker elicited by GABA(Aalpha2/3)-positive allosteric modulators at nonsedating anxiolytic doses. J Neurophysiol. 2015;113(1): 116-31.

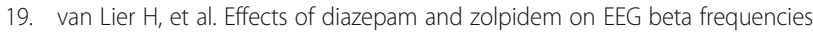
are behavior-specific in rats. Neuropharmacology. 2004;47(2):163-74.

20. Faulkner HJ, Traub RD, Whittington MA. Anaesthetic/amnesic agents disrupt beta frequency oscillations associated with potentiation of excitatory synaptic potentials in the rat hippocampal slice. Br J Pharmacol. 1999;128(8): 1813-25.

21. Kopell N, et al. Gamma rhythms and beta rhythms have different synchronization properties. Proc Natl Acad Sci U S A. 2000;97(4):1867-72.

22. Nutt DJ, Malizia AL. New insights into the role of the GABA(A)-benzodiazepine receptor in psychiatric disorder. Br J Psychiatry. 2001;179:390-6.

23. Haenschel C, et al. Gamma and beta frequency oscillations in response to novel auditory stimuli: A comparison of human electroencephalogram (EEG) data with in vitro models. Proc Natl Acad Sci U S A. 2000;97(13):7645-50.

24. Frohlich J, et al. Electrophysiological phenotype in Angelman syndrome differs between genotypes. Biol Psychiatry. 2019.

25. Scoles HA, et al. Increased copy number for methylated maternal 15q duplications leads to changes in gene and protein expression in human cortical samples. Mol Autism. 2011;2(1):19.

26. Parikshak NN, et al. Genome-wide changes in IncRNA, splicing, and regional gene expression patterns in autism. Nature. 2016;540(7633):423-7.

27. Urraca $\mathrm{N}$, et al. Significant transcriptional changes in $15 \mathrm{q}$ duplication but not Angelman syndrome deletion stem cell-derived neurons. Mol Autism. 2018;9:6.

28. Judson MC, et al. GABAergic neuron-specific loss of Ube3a causes angelman syndrome-like EEG abnormalities and enhances seizure susceptibility. Neuron. 2016;90(1):56-69.

29. Berrios J, et al. Loss of UBE3A from TH-expressing neurons suppresses GABA co-release and enhances VTA-NAc optical self-stimulation. Nat Commun. 2016;7:10702.

30. Egawa K, et al. Decreased tonic inhibition in cerebellar granule cells causes motor dysfunction in a mouse model of Angelman syndrome. Sci Transl Med. 2012;4(163):163ra157.

31. Ciarlone SL, et al. Effects of the synthetic neurosteroid ganaxolone on seizure activity and behavioral deficits in an Angelman syndrome mouse model. Neuropharmacology. 2017;116:142-50.

32. EM., M., Mullen scales of early learning : AGS edition. Circle Pines: American Guidance Service, 1995.

33. Scattone D, Raggio DJ, May W. Comparison of the Vineland Adaptive Behavior Scales, second edition, and the Bayley Scales of Infant and Toddler Development, third edition. Psychol Rep. 2011;109(2):626-34.

34. Oostenveld $\mathrm{R}$, et al. FieldTrip: open source software for advanced analysis of MEG, EEG, and invasive electrophysiological data. Comput Intell Neurosci. 2011;2011:156869.

35. A, H., Fast ICA for noisy data using Gaussian moments. (Vol. 5), Presented at the Circuits and Systems, 1999. ISCAS'99. Proceedings of the 1999 IEEE International Symposium on, IEEE, 1999. pp 57-61.

36. Jung TP, et al. Removal of eye activity artifacts from visual event-related potentials in normal and clinical subjects. Clin Neurophysiol. 2000;111(10): 1745-58.

37. Tallon-Baudry C, et al. Oscillatory gamma-band (30-70 Hz) activity induced by a visual search task in humans. J Neurosci. 1997;17(2):722-34.

38. Buzsaki G, Draguhn A. Neuronal oscillations in cortical networks. Science. 2004;304(5679):1926-9.

39. Delorme A, Makeig S. EEGLAB: an open source toolbox for analysis of single-trial EEG dynamics including independent component analysis. J Neurosci Methods. 2004;134(1):9-21.

40. Welch, P.D., The use of fast fourier transformfor the estimation of power spectra: a method based on time averaging over short, modified periodograms. IEEE transactions on audio and electroacoustics, 1967. AU-15(June). 
41. Barry RJ, et al. Electroencephalogram theta/beta ratio and arousal in attention-deficit/hyperactivity disorder: evidence of independent processes. Biol Psychiatry. 2009;66(4):398-401.

42. Jensen $\mathrm{O}$, et al. On the human sensorimotor-cortex beta rhythm: sources and modeling. Neuroimage. 2005;26(2):347-55.

43. Buzsaki G, Wang XJ. Mechanisms of gamma oscillations. Annu Rev Neurosci. 2012:35:203-25

44. Barker JL, McBurney RN. Phenobarbitone modulation of postsynaptic GABA receptor function on cultured mammalian neurons. Proc R Soc Lond B Biol Sci. 1979;206(1164):319-27.

45. Study RE, Barker JL. Diazepam and (--)-pentobarbital: fluctuation analysis reveals different mechanisms for potentiation of gamma-aminobutyric acid responses in cultured central neurons. Proc Natl Acad Sci U S A. 1981;78(11): $7180-4$

46. Mody I, et al. Bridging the cleft at GABA synapses in the brain. Trends Neurosci. 1994;17(12):517-25.

47. Shimono K, et al. Origins and distribution of cholinergically induced beta rhythms in hippocampal slices. J Neurosci. 2000;20(22):8462-73.

48. Conant $\mathrm{KD}$, et al. A survey of seizures and current treatments in $15 \mathrm{q}$ duplication syndrome. Epilepsia. 2014;55(3):396-402.

49. Arkilo $D$, et al. Electroencephalographic patterns during sleep in children with chromosome 15q11.2-13.1 duplications (Dup15q). Epilepsy Behav. 2016;57(Pt A):133-6.

50. Buzsaki G. Rhythms of the brain: Oxford University Press; 2006

51. Berg AT, et al. Adaptive behavior and later school achievement in children with early-onset epilepsy. Dev Med Child Neurol. 2013;55(7):661-7.

52. Papazoglou A, King TZ, Burns TG. Active seizures are associated with reduced adaptive functioning in children with epilepsy. Seizure. 2010;19(7):409-13.

53. Villarreal NW, et al. Adaptive skills and somatization in children with epilepsy. Epilepsy Res Treat. 2014;2014:856735.

54. Chapieski $L$, et al. Adaptive functioning in children with seizures: impact of maternal anxiety about epilepsy. Epilepsy Behav. 2005;7(2):246-52.

55. Moncla A, et al. Phenotype-genotype correlation in 20 deletion and 20 nondeletion Angelman syndrome patients. Eur J Hum Genet. 1999;7(2):131-9.

56. Lossie AC, et al. Distinct phenotypes distinguish the molecular classes of Angelman syndrome. J Med Genet. 2001;38(12):834-45.

57. Gentile JK, et al. A neurodevelopmental survey of Angelman syndrome with genotype-phenotype correlations. J Dev Behav Pediatr. 2010;31 (7):592-601.

58. Minassian BA, et al. Angelman syndrome: correlations between epilepsy phenotypes and genotypes. Ann Neurol. 1998:43(4):485-93.

59. Erickson CA, et al. Fragile $X$ targeted pharmacotherapy: lessons learned and future directions. J Neurodev Disord. 2017:9:7.

60. Berry-Kravis EM, et al. Drug development for neurodevelopmental disorders: lessons learned from fragile X syndrome. Nat Rev Drug Discov. 2018;17(4): 280-99.

61. Buxbaum JD, et al. Association between a GABRB3 polymorphism and autism. Mol Psychiatry. 2002;7(3):311-6.

62. Menold MM, et al. Association analysis of chromosome 15 gabaa receptor subunit genes in autistic disorder. J Neurogenet. 2001;15(3-4):245-59.

\section{Publisher's Note}

Springer Nature remains neutral with regard to jurisdictional claims in published maps and institutional affiliations.

Ready to submit your research? Choose BMC and benefit from:

- fast, convenient online submission

- thorough peer review by experienced researchers in your field

- rapid publication on acceptance

- support for research data, including large and complex data types

- gold Open Access which fosters wider collaboration and increased citations

- maximum visibility for your research: over $100 \mathrm{M}$ website views per year

At $\mathrm{BMC}$, research is always in progress.

Learn more biomedcentral.com/submissions 